\title{
RELATO DE CASO: INFARTO DO MIOCÁRDIO POR MIXOMA ATRIAL ESQUERDO
}

\author{
Henrique Rodrigues da Rocha MIGLIORI, Bernardo Borges MARQUES, Cláudio Magalhães RANGEL, \\ João Bosco de OLIVEIRA, Ana Lúcia Moulin Moreira de CARVALHO, Valdeon Caetano RODRIGUES \\ JUNIOR, Grazziela Vieira CIRQUEIRA, Arianne Alves COSTA, Nadia Arenas VERSALI, Bernardo Borges \\ MARQUES, Flávia de Almeida MIGUEZ \& Fernanda Nobre TORRES
}

Real e Benemérita Associação Portuguesa de Beneficência, São Paulo, Brasil.

*Autor para correspondência: analuciamoulin@yahoo.com.br

DOI: http://dx.doi.org/10.18571/acbm.133

\section{RESUMO}

Os tumores cardiovasculares primários são infrequentes, geralmente benignos e com alto potencial embólico. Objetivo: Relatar o caso de um paciente admitido para ressecção de mixoma atrial esquerdo que diante da realização do pré-operatório, evidenciou-se infarto do miocárdio prévio com provável etiologia embólica de fragmento tumoral. O caso a ser descrito corrobora com a literatura descrita sobre o tema pela raridade do mesmo. Metodologia: Relato de caso. Resultados: Ao eletrocardiograma observou-se área eletricamente inativa em parede inferior, o ecodopplercardiograma mostrou mixoma de átrio esquerdo com mobilidade, invadindo a cavidade ventricular na final diástole com a contração atrial esquerda, além de acinesia de parede inferior de ventrículo esquerdo e a cineangiocoronariografia não apresentou lesões coronarianas. Realizado exérese do mixoma atrial esquerdo sem intercorrências e o paciente foi encaminhado para consultas periódicas com ecodopplercardiograma para acompanhamento e tratamento da disfunção ventricular esquerda (sequela do evento isquêmico). Conclusão: Houve fortes indícios de infarto do miocárdio causado por êmbolo tumoral, pois o paciente já havia apresentado outro fenômeno embólico para o encéfalo, somando-se ao fato do exame de cineangiocoronariografia não ter demonstrado lesões coronárias obstrutivas que caracterizassem doença arterial coronariana.

Palavras chave: Mixoma atrial; Êmbolo tumoral; Infarto do miocárdio.

\begin{abstract}
Primary cardiovascular tumors are infrequent, usually benign and with high embolic potential. Objective: To report the case of a patient admitted for resection of left atrial myxoma who, prior to the preoperative period, showed a previous myocardial infarction with probable embolic etiology of a tumor fragment. The case to be described corroborates with the literature described on the subject by its rarity. Methodology: Case report. Results: The electrocardiogram showed an electrically inactive area in the inferior wall, doppler echocardiography showed a left atrial myxoma with mobility, invading the left ventricular cavity in the late diastole with left atrial contraction, as well as left ventricular inferior wall akinesia and the coronary angiography did not present coronary lesions. Performed excision of the left atrial myxoma without intercurrences and the patient was referred for periodic consultations with doppler echocardiography to follow up and treat left ventricular dysfunction (sequelae of the ischemic event). Conclusion: There were strong indications of myocardial infarction caused by tumor plunger, since the patient had already presented another embolic phenomenon to the encephalon, in addition to the fact that the coronary angiography did not demonstrate obstructive coronary lesions that characterized coronary artery disease.
\end{abstract}

Keywords: Atrial myxoma; Tumor plunger; Myocardial infarction. 


\section{Introdução}

Os tumores que acometem o músculo cardíaco constituem um grupo de neoplasia bastante raro, seja o diagnóstico feito clinicamente, seja feito por meio de necropsias. Até recentemente, o diagnóstico das neoplasias cardíacas era feito por meio de exames necroscópicos, na maioria dos casos (DALLAN, et al, 1988).

Os tumores cardíacos primários acometem todas as faixas etárias, com uma prevalência de $0,001 \%$ a $0,03 \%$ revelada em série de autópsias. Destes, os mixomas atriais são responsáveis por 30 a 50\% dos casos (BURKE e VIRMANI, 1996). O primeiro diagnóstico premortem de tumor primário do coração, um mixoma cardíaco, foi feito por Goldberg, em 1952 (BURKE e VIRMANI, 1996).

Ao considerarmos os tumores que acometem o músculo cardíaco, primários ou metastáticos, as principais formas de manifestação são as arritmias e os distúrbios de condução do sistema elétrico do coração. Fibrilação atrial, flutter atrial, extrasístoles ventriculares isoladas ou freqüentes, bloqueio de ramo direito (e de suas divisões) são fenômenos frequentemente observados nestes pacientes. Sinais e sintomas de insuficiência cardíaca congestiva são relatados e relacionados a tumores cardíacos que acometem as cavidades cardíacas direitas, preferencialmente. Na verdade, manifestam-se com sintomatologia de obstrução ao fluxo de sangue, necessitando diagnóstico diferencial com infarto, tromboses de veias supra-hepáticas, trombose de veias cavas superior, síndrome de compressão de veia cava superior, tumores do mediastino, estenose pulmonar, dentre outros (DALLAN, et al, 1988; LEÃO, et al, 1998; WIPPERMANN, et al, 2002 e GAUDRÉE, et al, 2000).

Os sinais e sintomas que são manifestados por um paciente com neoplasia cardíaca, em sua grande maioria, os tumores do coração e do saco pericárdico manifestam-se por sintomas que podem ser inerentes a inúmeras outras afecções não neoplásicas do sistema cardiovascular. Assim, a dor precordial, com ou sem alterações hemodinâmicas sistêmicas, deve ter seu diagnóstico diferencial com a insuficiência coronariana estabelecido. Arritmias, alterações da condução do estímulo cardíaco, focos de estimulação cardíaca ectópicos, distúrbios da condução do sistema Hiss-Purkinje e seus ramos, entre inúmeros outros sinais e sintomas, podem ser atribuídos a um grande número de cardiopatias adquiridas, dentre elas os tumores (primários ou secundários do coração e do saco pericárdico) (DALLAN, et al, 1988).

Com o advento, nas últimas décadas, de novos métodos complementares de diagnóstico está havendo aumento do número de pacientes que manifestam sintomas de neoplasias cardíacas e que podem ser tratados cirurgicamente. Métodos não invasivos e invasivos têm sido utilizados com baixa morbidade e alta eficácia no diagnóstico desta doença, propiciando o tratamento clínico (quimioterapia) ou cirúrgico (ressecção completa) dos tumores miocárdicos. Tais métodos diagnósticos (invasivos ou não) adquirem extrema importância no diagnóstico dos tumores do coração (CHAN, et al, 2001 e LEÃO, et al, 1998).

Neste estudo, relata-se o caso de um paciente com mixoma atrial esquerdo e infarto do miocárdio prévio com provável etiologia embólica de fragmento tumoral.

\section{Materiais e Métodos}

O relato de caso a ser descrito apresenta-se no campo da Saúde, na área de Cardiologia. A metodologia refere-se ao relato de caso, cujo paciente estudado é do sexo masculino, com 38 anos de idade, admitido no Hospital Real e Benemérita Associação Portuguesa de Beneficência para exérese de mixoma atrial esquerdo e que diante da realização do pré-operatório, evidenciou-se infarto do miocárdio prévio com provável etiologia embólica de fragmento tumoral. 


\section{Biomedica Brasiliensia}

Este relato de caso foi autorizado pela família do paciente para publicação, uma vez que a identificação do mesmo fosse preservada, seguindo os preceitos éticos da resolução 466/12 do Conselho Nacional de Saúde.

\section{Relato de Caso}

Paciente do sexo masculino, trinta e oito anos de idade, veio encaminhado ao serviço de cirurgia cardíaca do hospital Beneficência Portuguesa para exérese de mixoma atrial esquerdo. $\mathrm{O}$ mixoma atrial esquerdo foi diagnosticado após pesquisa para possível causa de acidente vascular encefálico com sequela de hemiplegia em dimídio direito, ocorrido há um ano e seis meses. $\mathrm{O}$ paciente apresentou também após seis meses do acidente vascular encefálico, único episódio de dor precordial atípica, de forte intensidade, quando então procurou um pronto socorro, foi medicado com analgésicos e obteve melhora da dor após algumas horas. Não houve demais investigações sobre o evento na ocasião.

Ao realizar o pré-operatório, evidenciou-se ao eletrocardiograma presença de onda $\mathrm{Q}$ em parede inferior (DII, DIII e AVf). Após o achado, foi solicitado ecodopplercardiograma que mostrou presença de massa móvel com pedículo localizado em teto de átrio esquerdo perto da desembocadura de uma das veias pulmonares medindo $43 \mathrm{~mm}$ x 21,6 $\mathrm{mm}$ e comprometimento da função global do ventrículo esquerdo com predomínio de acinesia de parede inferior do ventrículo esquerdo (FEVE\%: 0,49 Cubo).

A seguir, realizou-se cineangiocoronariografia a fim de investigação do evento isquêmico e a mesma não demonstrou lesões coronárias obstrutivas que caracterizassem doença arterial coronariana, o que sugeriu isquemia miocárdica de etiologia embólica.

Realizado então, exérese de mixoma atrial esquerdo sem intercorrências. Posteriormente, com boa evolução, o paciente obteve alta e foi encaminhado para consultas periódicas com ecodopplercardiograma para acompanhamento e tratamento da disfunção ventricular esquerda (sequela do evento isquêmico).

\section{Resultados}
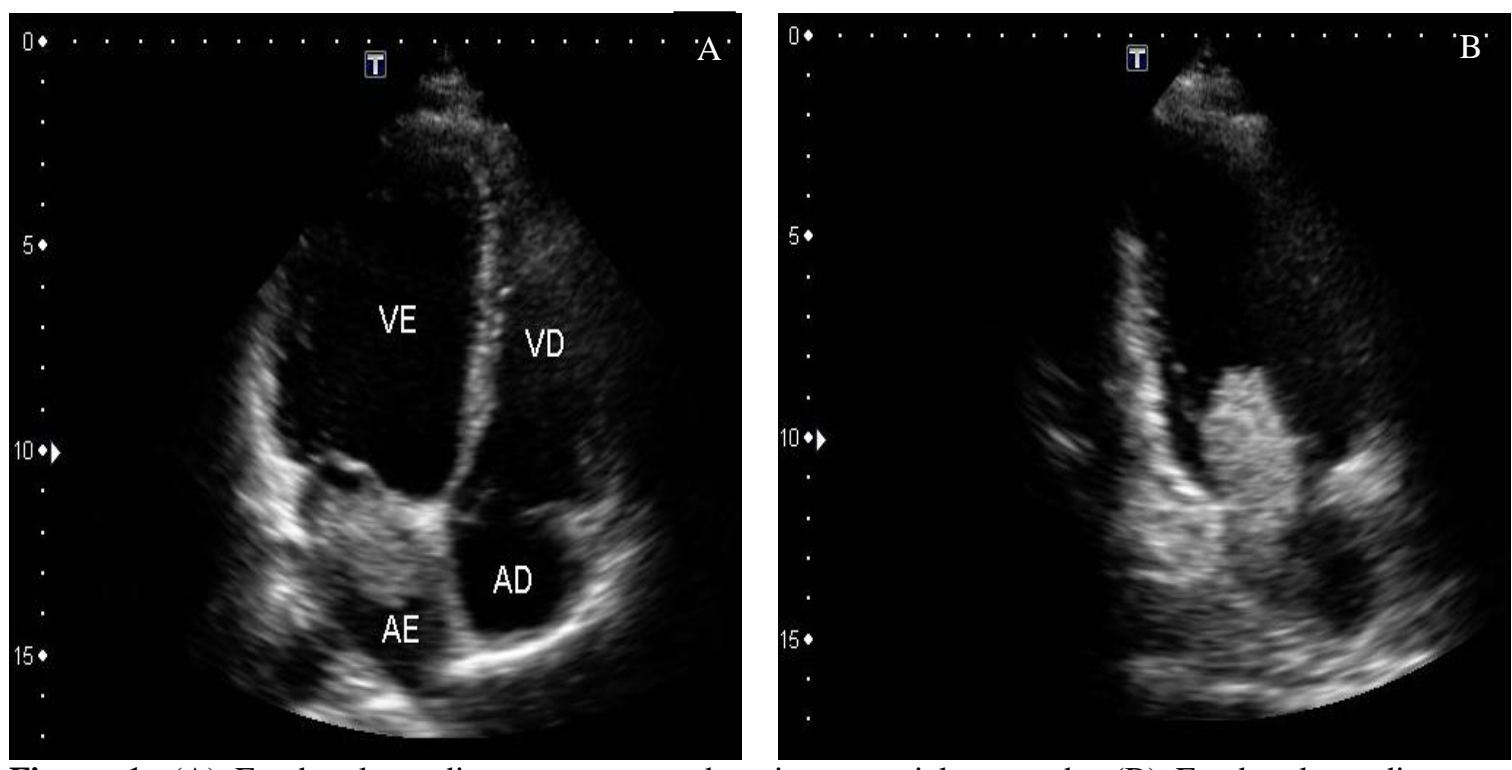

Figura 1: (A) Ecodopplercardiograma mostrando mixoma atrial esquerda. (B) Ecodopplercardiograma mostrando mobilidade de mixoma de átrio esquerdo invadindo cavidade ventricular na final diástole com a contração atrial esquerda. 


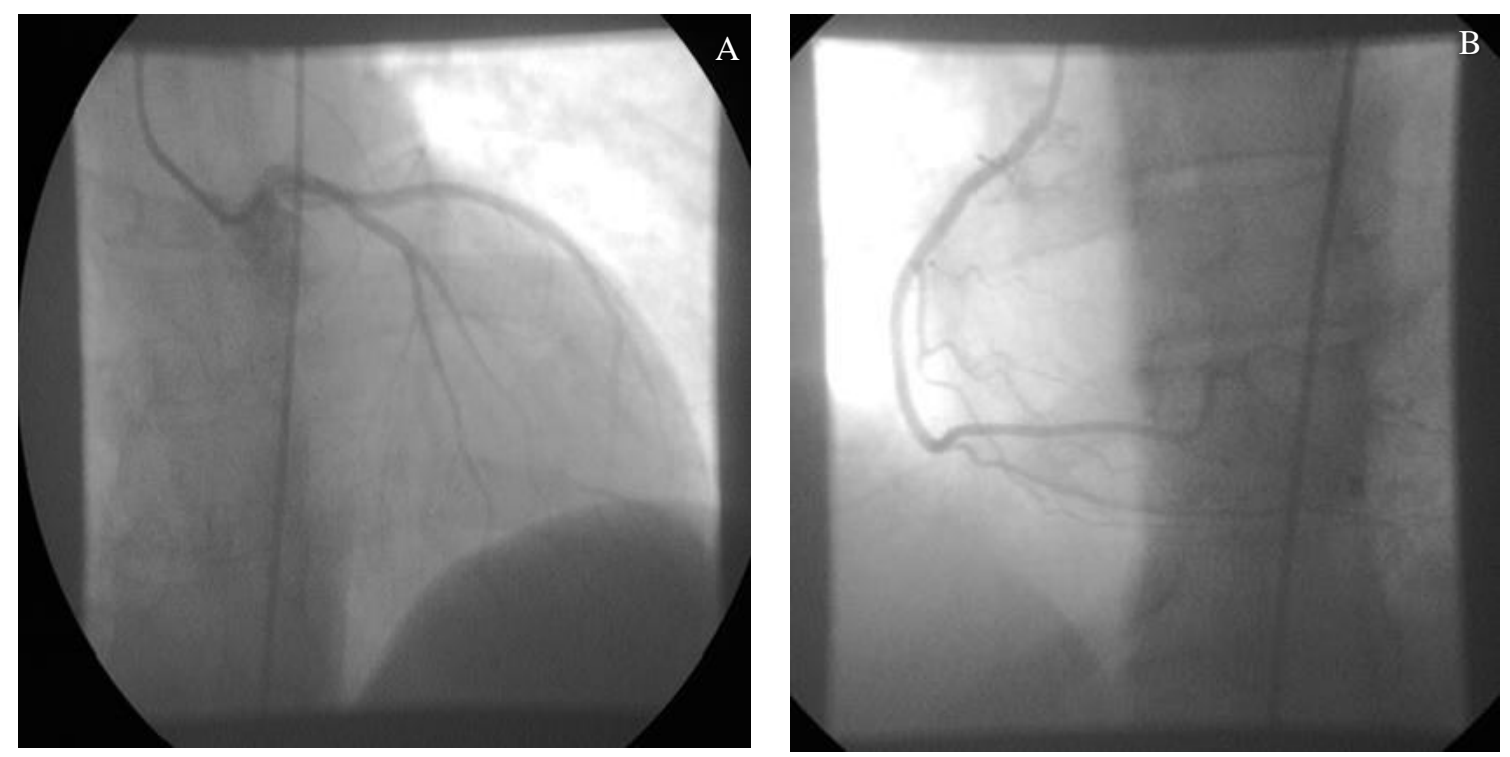

Figura 3: (A) Cineangiocoronariografia de artéria coronária esquerda em projeção oblíqua anterior direita, demonstrando ausência de lesões obstrutivas em seus ramos. (B) Cineangiocoronariografia de artéria coronária direita em projeção oblíqua anterior esquerda, demonstrando ausência de lesões obstrutivas em seus ramos.

\section{Discussão}

Os mixomas são os tumores primários cardíacos mais comuns, sendo que $83 \%$ deles ocorrem no átrio esquerdo, $12,7 \%$ ocorrem no átrio direito e 1,3\%, em ambos os átrios (BURKE e VIRMANI, 1996).

Apesar de vários relatos de casos documentados sobre metástases disseminadas, para vários locais anatômicos, o mixoma cardíaco típico é referido como uma neoplasia benigna, de uma forma convencional. Provavelmente, as metástases relatadas representam um crescimento tumoral, proveniente de fragmentos de tumor embólico, depositados na circulação de artérias em diferentes sítios remotos (LIBBY, et al, 2008).

A maioria dos pacientes se apresenta com, pelo menos, um dos sinais da tríade clássica de sintomas: obstrução cardíaca, embólica e manifestações estrutural-sistêmicas (PINEDE, et al, 2001).

A presença de embolização sistêmica em portadores de mixoma de átrio esquerdo é bem conhecida, embora a embolia coronária diagnosticada em vida seja extremamente rara. $\mathrm{Na}$ literatura disponível, a incidência em relato de autopsias é de 0,06\% (LEHRMAM, et al, 1985).

O ecodopplercardiograma constitui-se no exame com maior sensibilidade e especificidade para o diagnóstico das massas intracardíacas, principalmente nos mixomas atriais (KAYNAK, et al, 2001; SADEGHI, et al, 2002; FABRICIUS, et al, 2000 e GAUDRÉE et al, 2000). Em todos os casos relatados e aqui citados, a ecodopplercardiografia foi o método diagnóstico usado, revelando sua grande acurácia, mesmo sendo um método não-invasivo (ROBERTS, 1997). A cineangiocoronariografia não tem por finalidade o diagnóstico de mixoma cardíaco, se reservando apenas a pacientes com risco alto para doença arterial coronariana (LIBBY, et al, 2008).

O tratamento para o mixoma cardíaco é uma imediata ressecção cirúrgica do tumor, com os pacientes em circulação extracorpórea. A mortalidade pós-cirúrgica, imediata, na maioria das séries, vai de $0 \%$ a 7,5\% (PINEDE, et al, 2001). 


\section{Conclusão}

Houve fortes indícios de infarto do miocárdio causado por êmbolo tumoral, pois o paciente já havia apresentado outro fenômeno embólico para o encéfalo, o que causou acidente vascular encefálico isquêmico e sequelas do insulto (hemiplegia de dimídio direito). Houve também relato de único episódio de dor precordial atípica, além da presença de ondas Q em parede inferior (DII, DIII, AVf) ao eletrocardiograma e acinesia de parede inferior de ventrículo esquerdo ao ecodopplercardiograma que demonstraram presença de infarto do miocárdio prévio. Por fim, a cineangiocoronariografia não evidenciou lesões coronárias obstrutivas que caracterizassem doença arterial coronariana o que sugeriu infarto do miocárdio por êmbolo de fragmento tumoral como etiologia provável.

\section{Referências}

BURKE A, VIRMANI R: Fascicle 16, 3rd series: Tumors of the heart and the great vessels. In: Atlas of Tumor Pathology. Washington, DC, Armed Forces Institute of Pathology, 1996.

CHAN KL, VEINOT J, LEACH A, BEDARD P, SMITH S, MARQUIS JF. Diagnosis of left atrial sarcoma by transvenous endocardial biopsy. Can J Cardiol 2001; 17:206-8.

DALLAN LAO, POMERANTZEFF PMA, STOLF NAG. Tumores do coração. In: Raia AA, Zerbini EJ, editores. Clínica Cirúrgica Alípio Corrêa Neto. São Paulo: Sarvier; 1988. p 791-7.

FABRICIUS AM, AUTSCHBACH R, LOCHHAAS L, BROSE S, MOHR FW. Primary leftatrial leiomyosarcoma. Thorac Cardiovasc Surg 2000; 48:306-8.

GAUDRÉE L, CHABRUN A, CORBI P, LEVILLAIN P, HERPIN D. [Intracardiac metastasis of malignant melanoma. Literature review and case report]. Arch Mal Coeur Vaiss 2000; 93:1339-42.

KAYNAK K, BESIRLI K, ARSLAN C, OZGÜROGLU M, Oz B. Metastatic cardiac myxoma. Ann Thorac Surg 2001; 72:623-5.

LEÃO LEV, GIUDICE R, YAMASHITA HK, CONFORTI CA, CROTTI PLR, OLIVEIRA R. Thoracoscopic relief of symptomatic lipomatous hypertrophy of atrial septum. In: The $2^{\text {nd }}$ minimally invasive thoracic surgery interest group. International Symposium: Controversies in cardiothoracic surgery;1998;Hong Kong (China). Anais. Hong Kong (China):Minimally Invasive Thoracic Surgery Interest Group; 1998. p47.

LEHRMAM KL, PROZAN GB, ULLYOT D - Atrial myxoma presenting as acute myocardial infarction. Am Heart J 1985; 110: 1293-5.

LIBBY P, BONOW RO, MANN DL, ZIPES DP - Braunwald Heart's Disease: A Textbook of Cardiovascular Medicine, 8th edition. Saunders. 2008. vol.2.

PINEDE L, DUHAUT P, LOIRE R - Clinical presentation of left atrial cardiac myxoma. A series of 112 consecutive cases. Medicine (Baltimore) 80:159, 2001. 
ROBERTS WC - Primary and secondary neoplasms of the heart. Am J Cardiol 1997; 80: 6712.

SADEGHI N, SADEGHI S, KARIMI A. Mitral valve recurrence of a left atrial myxoma. Eur J Cardiothorac Surg 2002; 21:568-73.

WIPPERMANN J, ALBERS JM, BRANDES H, WAHLERS T. Redo-extirpation of a cardiac leiomyosarcoma to avoid transplantation. Thorac Cardiovasc Surg 2002; 50:62-3. 